\title{
The presentation and management of neonatal obstructive uropathies
}

\author{
J. H. JOHNSTON \\ F.R.C.S. \\ Alder Hey Children's Hospital, Liverpool
}

\section{Presentation}

The existence of a congenital urinary obstruction may be suggested when routine examination of the newborn infant reveals such signs as enlargement of one or both kidneys, distension of the bladder or slow, dribbling micturition. The paediatrician should also be alerted to the possibility of obstructive uropathy when there are present non-urological congenital anomalies which often secondarily involve the urinary tract or which are known commonly to co-exist with congenital urinary tract lesions.

Secondary involvement, with obstruction, of the urinary tract occurs with space-occupying masses in the pelvis such as hydrometrocolpos or a large intrapelvic component of a sacrococcygeal teratoma. The pelvic tumour, by displacing the bladder, leads to chronic urinary retention and upper tract dilatation. A congenital retroperitoneal teratoma may displace laterally, and obstruct, one or both ureters.

Examples of congenital anomalies which frequently coincide with congenital urological lesions include the following.

\section{(1) Anomalies of the umbilicus and umbilical cord}

A giant umbilical cord results from the accumulation of urine within it as a consequence of a urachoumbilical fistula. The condition may be an isolated anomaly but the possibility of an infravesical obstruction must be investigated. Absence of one umbilical artery is strongly suggestive of serious pathology in the alimentary and urinary tracts; Faierman (1960) found it to be present in $82 \%$ of cases of single umbilical artery. The baby with an exomphalos is very likely to have a urinary anomaly (McKeown, McMahon \& Record, 1953).

\section{(2) Abnormalities of the rectum and anus}

In the Alder Hey series (Johnston, 1969) urinary tract pathology was present in $51 \%$ of cases of high rectal anomaly and in $43 \%$ of cases with a low, anal lesion; these did not include examples of cloacal exstrophy. The corresponding figures in Smith's (1968) series were $38 \%$ and $24 \%$. Persistence of the original cloacal state in the female in which the urinary, alimentary and genital tracts share a common evacuatory channel is similarly frequently associated with upper urinary tract lesions.

\section{(3) Anomalies of the female genital tract}

Congenital absence of the vagina is associated with urinary tract anomalies in some $50 \%$ of cases (Bryan, Nigro \& Counsellor, 1949). A similar high incidence occurs with such conditions as duplication of the vagina and uterus but these may not be obvious clinically in the young child.

\section{(4) Deficient abdominal musculature}

Urinary tract anomalies of various degrees of severity are always present.

\section{(5) Cardiac anomalies}

Mehrizi (1962) found that $23 \%$ of 279 cases of malformation of the urinary tract had congenital heart disease. Ventricular septal defect was the most common lesion; of eighty-nine examples, twentytwo had urinary tract pathology.

\section{(6) Limb deformities}

Hanke \& Weicher (1965) reported that twenty-five of 100 children with phocomelia due to thalidomide embryopathy had urinary tract abnormalities. In the Marfan syndrome, arachnodactyly is commonly associated with cardiac and urinary anomalies; Loughridge (1959) recorded eight cases with the latter.

\section{(7) Exstrophy}

The more severe forms of exstrophy, particularly the cloacal type, carry a $50 \%$ incidence of upper urinary tract pathology (Soper \& Kilger, 1964).

\section{(8) Gonadal dysgenesis}

Turner's syndrome, which is suggested when the newborn infant exhibits neck webbing, a low hairline on the neck and oedema of the extremities, was associated with urinary lesions in $72 \%$ of cases in the series of Jeune et al. (1963). 
In a child with one of the above congenital anomalies urographic investigation of the urinary tract is advisable even in the absence of symptoms suggestive of urologic disease. Hypospadias, especially in its more severe forms, and undescent of the testis both carry a higher-than-average incidence of coincident urinary tract pathology but this is not sufficiently high to warrant routine urological investigation. Ascites in the newborn infant, particularly when associated with absent or abnormal voiding should arouse suspicion of the extravasation of urine into the peritoneal cavity as a consequence of a severe urinary obstruction; because of dialysis across the peritoneal membrane the urea content of the ascitic fluid is generally much lower than that of the urine.

\section{Pathology}

Urinary obstruction which originates post-natally characteristically leads to dilatation of the urinary tract above the obstructing lesion and to progressive thinning and destruction of the renal parenchyma as a result of back-pressure. Obstructions active during prenatal life may produce similar effects but often other pathological features exist. Prenatally obstructed ureters in addition to being dilated are often lengthened and tortuous and on occasions the resulting ureteral angulations may cause secondary obstructions, especially at the pelvi-ureteric junction. The kidneys may show, instead of, or in addition to, hydronephrotic atrophy, parenchymal changes indicative of failure of maturation; cysts of various sizes may be visible macroscopically, and on histological examination primitive glomeruli and tubules and nests of cartilage may be seen. The degree and extent of such developmental parenchymal dysplasia vary according to the severity of the obstruction and probably also according to its time of onset in foetal life. With severe obstructions of early onset the entire parenchyma may be grossly dysplastic and the kidney is then functionless (Fig. 1). Lesser degrees of dysplasia may, however, be found in kidneys showing useful functional activity.

\section{Clinical features and general management}

The most severe effects of urinary obstruction in the newborn infant are seen when the obstructive lesion is infravesical so that both kidneys are affected or when upper tract disease is bilateral or involves a solitary, or solitary functioning kidney. Such infants generally present with manifestations of azotaemia, acidaemia and, frequently, of urinary infection, often with complicating septicaemia. Anorexia, vomiting and diarrhoea are usually the initial symptoms; convulsions may occur and jaundice and a bleeding diathesis may develop as a result of septicaemia.

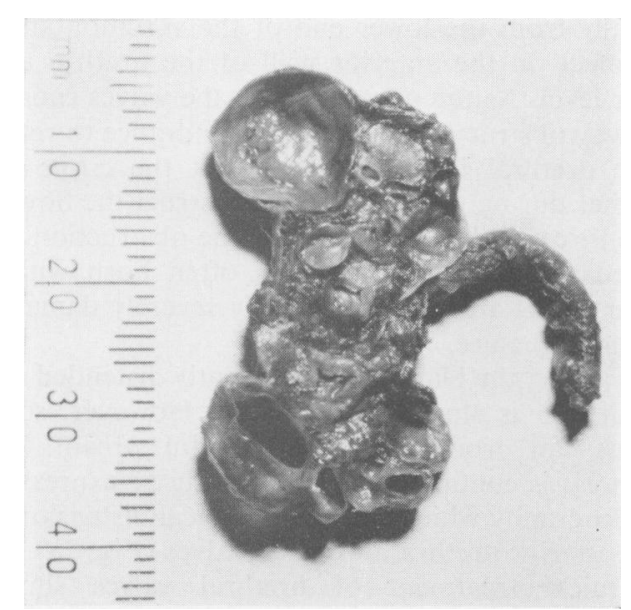

FIG. 1. Cystic, dysplastic, functionless (aplastic) kidney from newborn boy with posterior urethral valves.

Very often the first therapeutic needs of such seriously ill babies are the correction of severe oligaemic dehydration and of acidaemia by the appropriate intravenous infusions and the control of infection by chemotherapy. Pending the results of urine, and possibly of blood, culture, the most effective drug is likely to be ampicillin which can be given by intramuscular injection in a dosage of $\mathbf{5 0}$ $\mathrm{mg} / \mathrm{kg}$ body weight daily in four divided doses.

Peritoneal dialysis may be needed when the clinical or biochemical response to intravenous therapy is unsatisfactory and, in particular, in the presence of dangerous hypernatraemia or, less often, of hyperkalaemia. Commercially available packs (Dialafex, Allen and Hanbury; Dianeal, Baxter) are suitable for the newborn. Up to $50 \mathrm{ml}$ fluid/lb body weight is instilled into the peritoneal cavity at each cycle and siphoned out after $\frac{1}{2}-\frac{3}{4} \mathrm{hr}$.

When the child's condition has sufficiently improved, the appropriate urological investigations can be performed. In the presence of severely impaired renal function, intravenous urography requires a relatively high dose of contrast medium and delayed exposures for several hours after injection; prior dehydration of the infant by withholding feeds is unnecessary and may be dangerous. Endoscopy, with or without retrograde pyelography, and cystography with micturating or expression cystourethrography are commonly needed for diagnosis and full assessment.

\section{Posterior urethral valves}

In the male, the most severe form of congenital lower urinary tract obstruction is that caused by posterior urethral valves. In the commonest variety of this anomaly (Type 1 of Young \& McKay, 1929) the mucosal valve cusps diverge downwards and 
laterally from the lower end of the verumontanum and meet on the anterior wall of the urethra at a lower level. As the name implies, the valves cause a one-way obstruction; there is no hindrance to retrograde urethral instrumentation but the cusps fall together during micturition and obstruct the flow of urine through the urethra. Since the obstruction has existed prenatally, the child is often born with a urinary tract and kidneys already severely damaged by back-pressure.

As a rule, the bladder is persistently distended and micturition is slow and dribbling. However, some infants can produce quite a forceful stream. The diagnosis is confirmed by micturating or expression urethrography which shows the typical distension of the posterior urethra above the valves (Fig. 2a).

Surgical treatment of urethral valves should generally be directed firstly to the destruction of the valves themselves. This is best done by endoscopic diathermy coagulation; in the infant the endoscope must be inserted through a perineal urethrotomy. After operation, the urethral outline rapidly returns to normal (Fig. 2b) and complete bladder emptying occurs. In many instances, destruction of the valves is the only surgery required but, in others, there is impaired emptying of the dilated ureters into the bladder because of decompensation of the ureteral musculature. The resulting urinary stasis, with infection, is the commonest cause of failure of the child's condition to improve after the coagulation of the valves and requires urgent relief. This is best achieved by performing temporary, bilateral, high-loop cuta- neous ureterostomy. Through a small incision in the flank the ureter is brought to the surface in continuity, opened and sutured to the skin edges. The method provides free, tubeless urinary drainage and, in addition, allows the defunctioned ureter below the stoma to regain its muscular tone and contractility. When the child's general condition has improved sufficiently, which may require weeks or months, and when descending ureterography shows effective ureteral peristalsis and free emptying of the ureter into the bladder, the ureterostomies can be closed, one side at a time. On occasions, ureterostomy reveals that one kidney is functionless and in these circumstances nephrectomy is indicated.

An alternative method of dealing with the decompensated ureter has been described by Hendren (1970) who advocates a three or four-staged procedure by which both ends of both ureters are straightened and narrowed and the ureters are reimplanted into the bladder, employing an antireflux technique. In general, temporary loop ureterostomy is preferable in the seriously ill infant; Hendren's method is applicable to the less acute case. The indication for either type of ureteral surgery must, however, be based on the clinical state of the child and not merely on the radiological appearance of the urinary tract since severely dilated and tortuous ureters can straighten remarkably with time simply by destruction of the valves (Johnston \& Kulatilake, 1972).

Less common causes of infravesical obstruction in the male infant include anterior urethral valve,

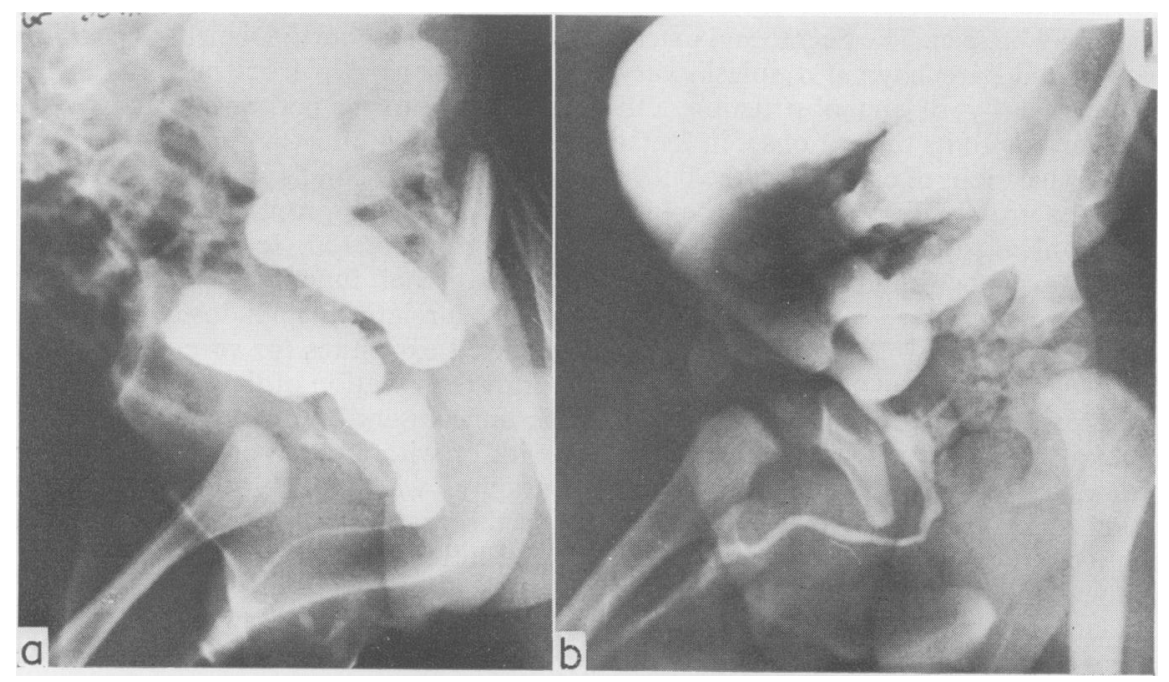

Fig. 2. Cysto-urethrograms in infant with posterior urethral valves. (a) Pre-operative. Severe dilatation of prostatic urethra. Reflux to very dilated ureter. (b) Six months after endoscopic diathermy coagulation of valves. No residual urethral obstruction. 
posterior urethral diaphragmatic stenosis (Young's Type 3 valves) and posterior urethral polyp. In general, the management of such cases is similar to that of posterior urethral valves; that is, the primary pathology is treated initially and secondary pathological changes in the ureters are dealt with, if necessary, either by temporary ureterostomy or by ureteral reconstruction.

\section{Hydrocolpos}

In the female infant, severe degrees of intrinsic infravesical obstruction are rare. More often, impaired bladder emptying results from extrinsic compression. The commonest cause of this is hydrocolpos resulting from an imperforate hymen or from an occluding vaginal septum at a higher level. The normal prenatal mucoid secretions which result from stimulation of the cervical and vaginal glands by maternal hormones, accumulate above the obstructing lesion so that the vagina and, to a lesser degree, the uterus become distended with mucinous fluid. The pelvic mass displaces the bladder upwards into the abdomen leading to incomplete bladder emptying and upper urinary tract dilatation. When the obstructing membrane is low in the vagina the diagnosis is usually readily made by inspection of the vulva which reveals a bulging septum (Fig. 3); with a higher vaginal occlusion, vaginoscopy may be needed. The obstruction of the genital and urinary tracts is relieved by incision of the obstructing membrane and release of the accumulated secretions. With

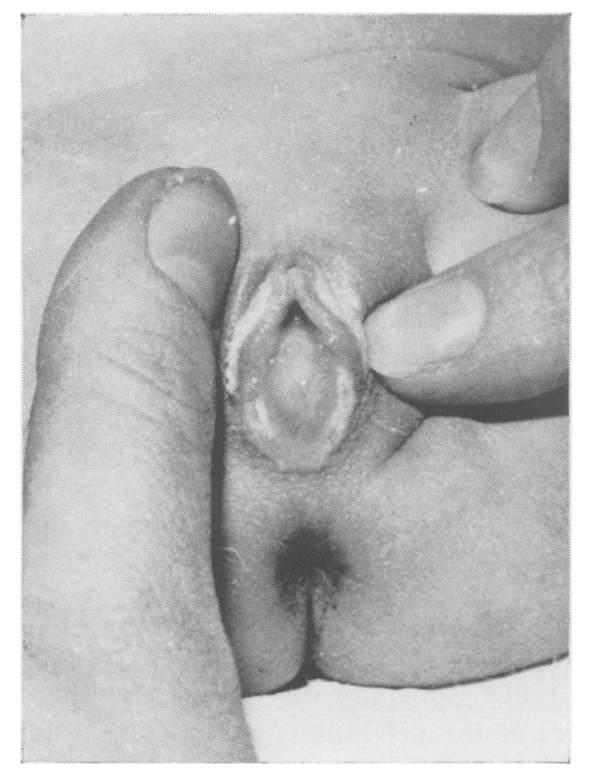

Fig. 3. Vulva of newborn girl with hydrocolpos. Bulging membrane at vaginal introitus. a very high vaginal obstruction, this may necessitate laparotomy; the distended vagina is opened on its anterior wall and the membrane is incised from above, with guidance from below. As a rule, the urinary tract obstruction in these babies has developed fairly late in prenatal life and the dilated upper urinary tract generally shows good and prompt improvement when the vaginal obstruction has been relieved.

\section{Ectopic ureterocele}

An ectopic ureterocele is a cystic dilatation of the intravesical and intraurethral portions of a ureter draining the upper moiety of a double kidney. The twin ureters both penetrate the bladder musculature at about the usual site. The ureter from the lower hemikidney opens into the bladder; the other continues under the vesical mucosa to open ectopically in the proximal urethra. The ectopic ureter dilates as a consequence of stenosis of its orifice or as a result of compression by the enclosing vesico-urethral sphincter musculature. The condition affects both males and females but is commoner in the latter.

In some instances the pathological changes are restricted to the ectopic ureter and its related renal parenchyma but frequently the ureterocele itself causes secondary obstructive effects. The twin ureter may be obstructed by the ureterocele encroaching upon and compressing its intravesical part (Fig. 4) or the upper urinary tract may be affected bilaterally when the ureterocele obstructs the urethra and interferes with bladder emptying (Fig. 5). Occasionally,



FIG. 4. Operation photograph showing large left ectopic ureterocele in bladder of baby girl. The probe is in the orifice of the twin ureter which was obstructed by the ureterocele. 




FIG. 5. Intravenous pyelogram in baby girl with left ectopic ureterocele which obstructed the twin ureter and the vesical outlet. The left upper hemikidney draining into the ureterocele does not concentrate the contrast medium. The left lower hemikidney is severely hydronephrotic, and the right side shows moderate hydroureteronephrosis. The bladder is distended and the ureterocele is seen as a filling defect in the base.

in girls, acute urinary retention may be precipitated by the ureterocele prolapsing through the urethra to the exterior and becoming strangulated.

As with other forms of congenital urinary obstruction, the more severe the degree of obstruction the earlier the child presents, and as a rule it is cases in which the ureterocele blocks the urethra that are seen most frequently in the neonatal period.

Relief of the urinary obstruction is obtained by decompression of the ureterocele. Simple endoscopic incision of the ureterocele wall may rarely be required in the seriously ill baby with severe infection but definitive surgery requires an open, transvesical operation and uncapping or de-roofing of the ureterocele. The ureterocele floor remains in situ. It is important to remove the projecting part of the ureterocele completely, especially in its urethral portion, otherwise folds remain which may obstruct micturition. Surgery to the ipsilateral twin ureteral orifice or to the contralateral orifice may be needed in order to cure vesico-ureteric reflux. The parenchyma of the upper hemikidney draining into the ureterocele has, in the majority of cases, been irreparably damaged and rarely shows significant improvement after the ureterocele has been removed; as a rule, removal of this hemikidney and its ureter are required. Secondarily obstructed twin and contralateral ureters and renal segments are, however, capable of considerable anatomical and functional improvement.

\section{Obstructed mega-ureter}

In this condition there is a functional obstruction in the lower portion of the ureter. There is, as a rule, no anatomical ureteral narrowing but an aperistaltic segment exists which interferes with the flow of urine. The precise pathogenesis of the condition remains obscure. There is an obvious similarity to Hirschsprung's disease of the large bowel but ganglion cells are stated to be present normally throughout the ureter (Bischoff \& Busch, 1961). Murnaghan (1957) found an abnormality in the ureteral musculature in that there is an excessive number of circular fibres in proportion to longitudinal ones. The obstruction leads to ureteral dilatation and back-pressure atrophy of the renal parenchyma. Infection commonly complicates the lesion and calculi may form in the dilated ureter and kidney. The obstructed megaureter is not commonly encountered in the neonatal period but it may be when the condition involves a solitary kidney or, less often, when it is bilateral.

Surgical relief of the obstruction requires that the ureter above the obstructing segment is re-implanted into the bladder. Very often ureteral tortuosities must be freed and a redundant portion of the ureter resected. The extremity of the remaining ureter is then narrowed and re-implanted into the bladder to lie in a submucous tunnel so that subsequent vesicoureteric reflux will be prevented.

The obstructed mega-ureter has great capacity for improvement when the obstruction has been relieved and quite severely dilated pyelons can return to virtually normal calibre with a corresponding improvement in renal function (Fig. 6).

\section{Pelvic hydronephrosis}

Congenital obstruction of the pelvi-ureteric junction may result from a variety of causes. The most common lesions are firstly, an angulation of the ureter on the pelvis resulting from a high ureteral origin and secondly, a ureteral kinking caused by an accessory or aberrant artery crossing to the lower pole of the kidney. Less frequently encountered conditions include a mucosal flap-valve covering the orifice of the ureter in the pelvis and the persistence, in exaggerated form, of foetal ureteral folds. It has often been contended that the gross pathological changes at the pelvi-ureteral junction, although in themselves obstructive, are in most instances secondary to a functional disorder of peristaltic continuity; pressure-flow studies of hydronephrotic 


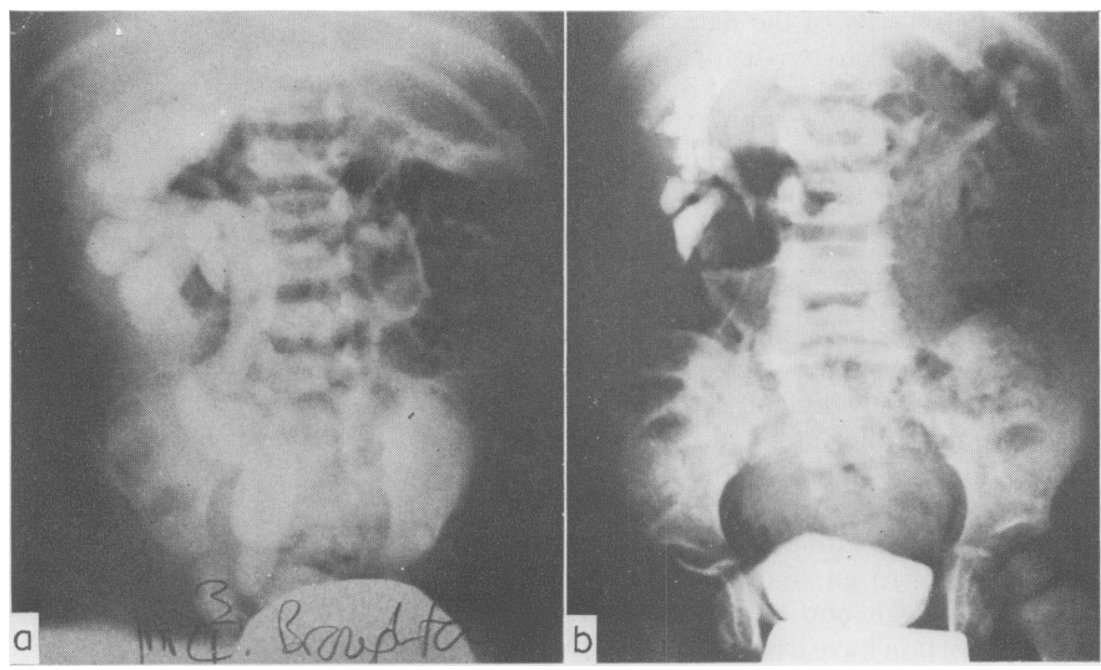

FIG. 6. Intravenous pyelograms of baby boy with obstructed mega-ureter affecting a solitary right kidney. (a) Before surgery. Ureter dilated and tortuous. Severely dilated calyces. (b) Two years after ureteral re-implantation into bladder. Ureter of normal calibre. Improvement in calyceal dilatation.

kidneys in children have, however, indicated that a functional obstruction is an unusual occurrence (Johnston, 1969).

When pelvic hydronephrosis presents in early infancy, the condition commonly proves to be bilateral or to affect a solitary or solitarily functioning kidney. In the series of twenty-six infantile cases of Williams \& Karlaftis (1966), ten were bilateral and in five the opposite kidney was afunctional. Contralateral renal agenesis may be present (Fig. 7) but a more common contralateral lesion is multicystic renal dysplasia associated with ureteral atresia. In five of the author's series of twenty-two cases of multicystic dysplasia the contralateral kidney was the site of a hydronephrosis. In such cases the multicystic kidney is usually the presenting clinical feature, a lobulated,freely movable and often transilluminable loin swelling being found on routine neonatal examination. Very often the hydronephrotic kidney itself forms a palpable mass and urinary infection is commonly present.

In the seriously ill infant with an infected hydronephrosis involving a solitary functioning kidney, nephrostomy may be needed as an emergency measure (Fig. 7). Definitive reconstructive surgery for the relief of the obstruction does not differ from that performed in the older child. In most instances the Anderson-Hynes (Anderson, 1953) dismembered pyelo-ureteroplasty is very effective.

\section{Prognosis}

The correction of fluid and electrolyte disorders consequent upon renal insufficiency, the prompt relief of obstruction and the control of complicating infection are of obvious immediate prognostic importance in cases of neonatal urinary obstruction but the long-term outlook for the child with a congenital obstruction affecting all his renal tissue

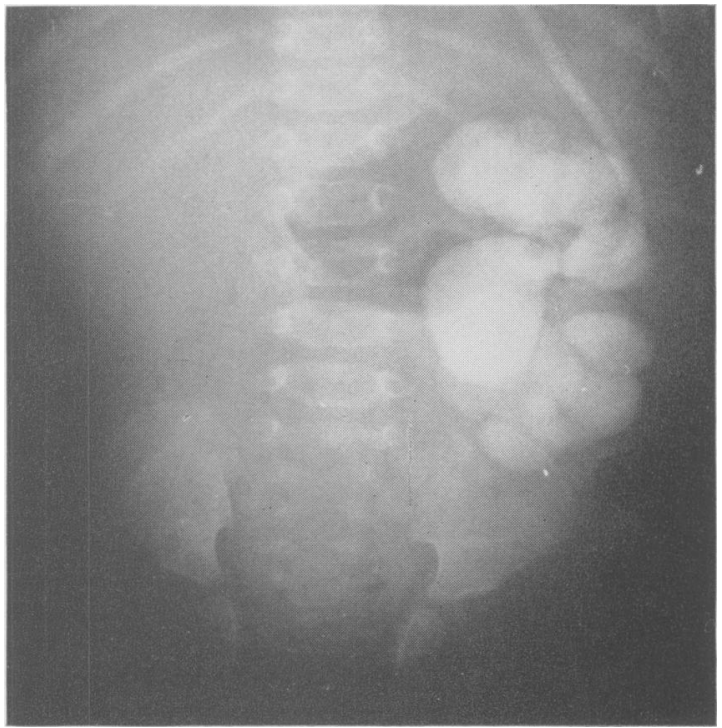

FIG. 7. Pelvic hydronephrosis affecting solitary left kidney in infant boy. Emergency nephrostomy has been performed. 
depends mainly upon the severity of the damage inflicted upon the kidney parenchyma before birth. The degree of this varies with the degree of obstruction and with its time of onset in foetal life. With an early, severe obstruction such as often occurs with posterior urethral valves, the kidneys may be incapable of sustaining life. In the author's series of sixty-two cases of posterior urethral valves, twentyfour boys presented in the first month of life; the mortality rate with these, over a maximal follow-up of 12 years, has been $50 \%$. At the outset, however, prognostication can be extremely difficult. The initial azotaemia and acidaemia in the infant are in part the result of dehydration and infection and are, of course, exaggerated by the immaturity of function in the newborn kidney. All infants with urinary obstruction are entitled, therefore, to an optimistic therapeutic approach. Even though one must be aware that a considerable proportion have irreparable renal damage, many others just as ill at the time of presentation have kidneys which can respond to the relief of urinary obstruction and these have a good longterm outlook.

\section{References}

ANDERSON, J.C. (1953) Hydronephrosis. In: Modern Trends in Urology (Ed. by E. Riches). Butterworths, London.

Bischoff, P.F. \& Busch, H.G. (1961) Origin, clinical experiences and treatment of urinary obstructions of the lower ureter in childhood. Journal of Urology, 85, 739.

Bryan, A.L., Nigro, J.A. \& Counsellor, V.S. (1949) One hundred cases of congenital absence of vagina. Surgery, Gynecology and Obstetrics, 88, 79.
FaIerman, E. (1960) The significance of one umbilical artery. Archives of Disease in Childhood, 35, 285.

HANKE, H. \& WeICKER (1965) Thalidomide embryopathies. Renal malformations. Deutsche medizinische Wochenschrift, 90, 2200.

HeNDREN, W.H. (1970) A new approach to infants with severe obstructive uropathy: Early complete reconstruction. Journal of Pediatric Surgery, 5, 184.

Jeune, M., Bertrand, J., Deffrerne, R. \& Forget, M. (1963) Frequency of renal anomalies in Turner's syndrome in children. Annals of Endocardiology, 24, 856.

Johnston, J.H. (1969) In: Neonatal Surgery (Ed. by P. P. Rickham and J. H. Johnston). Butterworths, London.

JoHNSTON, J.H. (1969) The pathogenesis of hydronephrosis in children. British Journal of Urology, 41, 724.

Johnston, J.H. \& Kulatilake, A.E. (1971) The sequelae of posterior urethral valves. British Journal of Urology, 43, 743.

Loughridge, L.W. (1959) Renal abnormalities in Marfan syndrome. Quarterly Journal of Medicine, 28, 531.

McKeown, T., McMahon, B. \& Record, B.G. (1953) An investigation in sixty-nine cases of exomphalos. American Journal of Human Genetics, 5, 168.

Mehrizi, A. (1962) Congenital malformation of the heart associated with congenital anomalies of the urinary tract. Journal of Pediatrics, 61, 582.

MuRnaghan, G.F. (1957) Experimental investigation of the dynamics of the normal and dilated ureter. British Journal of Urology, 29, 403.

SMITH, E.D. (1968) Urinary anomalies and complications in imperforate anus and rectum. Journal of Pediatric Surgery, 3, 337.

SOPER, R.T. \& Kilger, K. (1964) Vesico-intestinal fissure. Journal of Urology, 92, 490.

Williams, D.I. \& Karlaftis, C.M. Hydronephrosis due to pelvi-ureteric obstruction in the newborn. British Journal of Urology, 38, 138.

Young, H.H. \& McKAY, R.W. (1929) Congenital valvular obstruction of prostatic urethra. Surgery, Gynecology and Obstetrics, 48, 509. 